\title{
Demo Abstract: Data Collection in Delay Tolerant Mobile Sensor Networks using SCAR
}

\author{
Cecilia Mascolo \\ Dept. of Computer Science, \\ University College London \\ Gower Street, London \\ WC1E 6BT, United Kingdom \\ c.mascolo@cs.ucl.ac.uk
}

\author{
Mirco Musolesi \\ Dept. of Computer Science, \\ University College London \\ Gower Street, London \\ WC1E 6BT, United Kingdom \\ m.musolesi@cs.ucl.ac.uk
}

\author{
Bence Pásztor \\ Dept. of Computer Science, \\ University College London \\ Gower Street, London \\ WC1E 6BT, United Kingdom \\ b.pasztor@cs.ucl.ac.uk
}

\section{Categories and Subject Descriptors}

C.2.1 [Computer-Communication Networks]: Network Architecture and Design - wireless communication, store and forward networks, distributed networks; C.2.2 [ComputerCommunication Networks]: Network Protocol—routing protocols

\section{General Terms}

Design, Algorithms, Experimentation

\section{Keywords}

Sensor networks, delay tolerant networks, mobile sinks, adaptive routing, buffer management, time series forecasting

\section{OVERVIEW AND MOTIVATION}

Sensor devices are now present in or attached to virtually any sorts of items, from vehicles and furniture to humans and animals. This generates networks of wireless connected devices with topologies which could be very dynamic. The monitoring abilities of these devices range from pollution and temperature to health-care and mobility. The amount of data generated by these applications is usually quite large, however, luckily, the data is also, in most cases, delay tolerant, in the sense that it can be stored in the network for a certain period of time before being collected.

The scenario we envisage in this demonstration is one where mobile sensor nodes (e.g., animals, vehicles or humans) route data through each others in order to reach sink nodes, which can be either mobile or fixed. The fixed nodes are intended as nodes connected to a backbone network and therefore able to forward the data to the appropriate destination. The challenges offered by this scenario are many and include the quantity of data to be shipped to the sinks, the potentially scarce communication power (i.e., energy and bandwidth) of the nodes, the possible communication and sensor hardware faults, the mobility and the scarce buffer size of the nodes.

Different techniques could be employed for mobile sensor data gathering. A basic strategy would be to only allow data delivery when sensors are in direct proximity of the sinks. This technique has very little communication

Copyright is held by the author/owner(s).

SenSys'06, November 1-3, 2006, Boulder, Colorado, USA.

ACM 1-59593-343-3/06/0011. overhead, given that messages are only sent directly from the sensor node generating messages to the sink. However, depending on how frequently sensor nodes meet the sinks, the delivery of the data might be very poor. This is particularly true if the sinks are very few and spread out. More refined techniques include epidemic-style approaches [6], which spread the data over the sensor network, so that eventually a sink could be reached. This approach has very good delivery ratio if buffers are sufficiently large, however the overhead in terms of communication and, therefore, energy is quite high. Some solutions have been presented in literature, including the use of data mules with scheduled routes [3].

For these reasons, the spreading of the messages needs to be carefully controlled and traded off for the delivery ratio. This is even more true if the nodes have limited memory so that the buffer size is small and only few messages can be stored. Moreover, we consider scenarios where the routes of the potential message carriers may not be known a priori, as in the case of systems where devices attached to animals or humans are exploited to deliver the data to the sinks.

Starting from these considerations, we have developed SCAR (Sensor Context-aware Adaptive Routing) [5], a routing approach which uses prediction techniques over context of the sensor nodes (such as previously co-location with the sinks, battery level, etc.) to foresee which of the sensor neighbors are the best carriers for the data messages. We further adopt different classes of messages in order to achieve an intelligent buffer management. In this demonstration, we will show the feasibility of the implementation of this protocol, based on Kalman filter prediction techniques [1]. We will also prove that our protocol is able to deliver the data to the sinks without the a priori knowledge of the routes of the carriers.

We now first introduce the protocol, then we give an outline of its implementation on sensors running Contiki [2]. We will then describe the setting of the actual demonstration.

\section{SCAR ROUTING}

We now provide the key concepts of the protocol. These are useful to appreciate the technical contribution of our demonstration. The complete description of the protocol is presented in [5].

The decision process by which nodes select the best carriers is based on prediction of the future evolution of the system. Our solution relies on the analysis of the history of the movement pattern of the nodes and their co-location 
with the sinks and on the evaluation of the current available resources of the sensors. In particular, each node evaluates its change rate of connectivity, co-location with sinks, and battery level. The forecasted values of the context attributes describing the context are then combined to define a delivery probability $P\left(s_{i}\right)$ for each sensor $s_{i}$ to deliver bundles to sinks.

While moving, the sensors will transfer their data to other sensors only if these have a higher probability to deliver the data to sinks (i.e., they are better carriers). The calculation of the delivery probability is local and it does not involve any distributed computation. Nodes exchange information about their current delivery probability and their available buffer space with the neighbors only periodically.

\section{PROTOTYPE IMPLEMENTATION}

We have implemented SCAR on the Tmote Sky nodes using the Contiki Operating System [2]. The Kalman Filter predictor has been implemented to allow decision making on forwarding and buffering. As shown in the demonstration, our implementation is very lightweight.

The sensors are equipped with low-power microprocessors. As these are simple, 16-bit processors (TI MSP430), they do not provide floating-point support, which is essential for the calculation of the Kalman predictions. To enable the algorithm to work, a software emulation had to be used. The 16-bit processor limits the accuracy of the calculations; however, for the purpose of the predictions, this is acceptable. The algorithm is implemented using protothreads running in the Contiki kernel. Each thread is given a task, such as calculating and sending the predictions of the sensor, receiving other motes' information (routing messages) and storing it in a table, and sending and receiving the actual messages. The threads are executed periodically (in case of routing messages), or when necessary (if there is a message in the buffer to send). The messages are sent as UDP packets, using Contiki libraries. Each mote is given a unique IP address during set-up. Messages are stored in a buffer as C structures. Information about other sensors are also stored as $\mathrm{C}$ structures, and updated every time a new routing message is received. Also, when a message reaches the sink the message with the path followed (i.e., the IP addresses of the sensors traversed) is displayed, and deleted from the buffer.

\section{SCENARIO AND DEMO DESCRIPTION}

The aim of this demonstration is to show how the protocol is able to select the right carriers for the data generated by the sensors and then to deliver them to the sinks. As we have explained, the choice of the carrier(s) is based on information related to battery power, connectivity and buffer space of the sensors. The setting of the demonstration is shown in Figure 1 will be as follows:

- T-mote Sky $A$, acting as a data (temperature) source, will be placed in a corner (far from the demonstration booth) of the demonstration hall;

- T-mote Sky B, connected to a laptop through USB, acting as a sink and will be placed on the booth table; sensors $A$ and $B$ are not in reach of each other, as indicated in the picture; the laptop will act as a display for the received data;

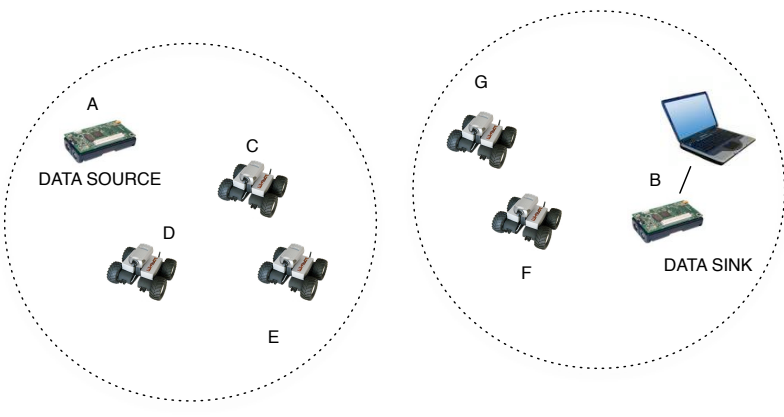

Figure 1: Demonstration Topology.

- 5 T-Motes Skys attached to people or on Lego MindStorms; these will represent the mobile carriers.

These sensors will be characterized by different battery level and different patterns of mobility and connectivity with the sink $B$. One of the scenarios that will be demonstrated is the following: sensors $C, D, E$ are often in reach of the source $A$, while $F$ and $G$ are not and, instead, they are often in reach of sink $B$; initially, only sensor $C$ has a full buffer, while sensor $D$ and $E$ do not, later also the buffer on sensor $D$ will fill up. Sensor $F$ has high battery levels while sensor $G$ has low power. The demonstration will show how initially node $A$ running SCAR will select nodes $E$ and/or $D$ as first hops, while later in time, it will only select $C$. The next hop for the data then will be $F$ as it has higher battery. From $F$ the data has a chance to reach $\operatorname{sink} B$ as $F$ is frequently roaming towards $B$. When the data reaches the sink, a message showing the path the message took, the time, and the temperature value, will be displayed. We also will show the times related to the decision making process guided by the Kalman filters in the different stages.

Acknowledgments: We acknowledge the support of EU IST project RUNES, EPSRC Project CREAM and of the Nuffield Foundation.

\section{REFERENCES}

[1] P. J. Brockwell and R. A. Davis. Introduction to Time Series and Forecasting. Springer, 1996.

[2] A. Dunkels, B. Gronvall, and V. Thiemo. Contiki - a lightweight and flexible operating system for tiny networked sensors. In Proceedings of the First IEEE Workshop on Embedded Networked Sensors, Tampa, FL, November 2004.

[3] D. Jea, A. A. Somasundara, and M. B. Srivastava. Multiple Controlled Mobile Elements (Data Mules) for Data Collection in Sensor Networks. In DCOSS, pages 244-257, 2005.

[4] R. L. Keeney and H. Raiffa. Decisions with Multiple Objectives: Preference and Value Tradeoffs. Wiley, 1976.

[5] C. Mascolo and M. Musolesi. SCAR: Context-aware Adaptive Routing in Delay Tolerant Mobile Sensor Networks. In Proceedings of the Delay Tolerant Networks Symposium. ACM International Wireless Communications and Mobile Computing Conference (IWCMC) 2006. ACM Press, July 2006.

[6] A. Vahdat and D. Becker. Epidemic routing for partially connected ad hoc networks. Technical Report CS-2000-06, Department of Computer Science, Duke University, 2000.

[7] Y. Wang and H. Wu. DFT-MSN: The Delay/Fault-Tolerant Mobile Sensor Network for Pervasive Information Gathering. In Proceedings of INFOCOM'06, Barcelona, Spain, April 06. 